\title{
Host range of meliolaceous fungi in India
}

\section{V.B. Hosagoudar ${ }^{1} \&$ G.R. Archana ${ }^{2}$}

1,2 Tropical Botanic Garden and Research Institute, Palode, Thiruvananthapuram, Kerala 695562, India Email: ${ }^{1}$ vbhosagoudar@ rediffmail.com

Date of online publication 26 May 2009 ISSN 0974-7907 (online) | 0974-7893 (print)

Editor: Richard Mibey

\section{Manuscript details:}

Ms \# 02061

Received 13 September 2008

Final received 07 April 2009

Finally accepted 15 May 2009

Citation: Hosagoudar, V.B. \& G.R. Archana (2009). Host range of meliolaceous fungi in India. Journal of Threatened Taxa 1(5): 269282

Copyright: (c) V.B. Hosagoudar \& G.R. Archana 2009. Creative Commons Attribution 3.0 Unported License. JoTT allows unrestricted use of this article in any medium for nonprofit purposes, reproduction and distribution by providing adequate credit to the authors and the source of publication.

Author Details: V.B. HoSAgOUdAR has more than three decades of research experience, has published five books on fungi including Monographs on Meliolales of India and has authored more than 300 research publications. G.R. ARCHANA is currently working on foliicolous fungi of Western Ghats region of Kerala state under the guidance of Dr. V.B. Hosagoudar. She has 22 research papers in national and international journals.

Author Contribution: The study: GRA with the guidance of $\mathrm{VBH}$ for the higher studies on foliicolous fungi. The current paper: Written by both authors.

Acknowledgements: We thank Dr. S Ganeshan, Director, Tropical Botanic Garden and Research Institute, Palode, Thiruvananthapuram, Kerala for the facilities.
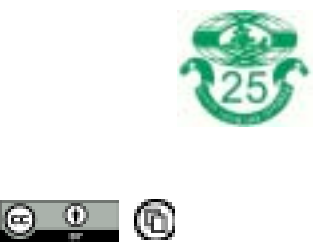

OPEN ACCESS |FREE DOWNLOAD
Abstract: The order Meliolales comprises two families, namely, Armatellaceae and Meliolaceae. Except the genera Endomeliola and Pauhia, India represents rest of the nine genera of this group. The family Armatellaceae includes two genera, namely, Armatella and Basavamyces. The family Meliolaceae includes seven genera: Amazonia, Appendiculella, Asteridiella, Ectendomeliola, Irenopsis, Meliola and Prataprajella. All these nine genera represent 613 species and infraspecific taxa known till the year 2006, infected 766 host plants belonging to 349 host genera distributed among 104 families. All the host families and the fungal genera are arranged alphabetically with their corresponding parasite and the host plant. The corresponding number after the host family represents the number of meliolaceous taxa known on the members of that family.

Keywords: Families, hosts, India, Meliolaceae

Black colony forming parasitic fungi are known as "Black or dark mildews". These are obligate ecto-parasites producing black colonies on the surface of the host plants. The black colony forming parasitic fungi belong to several taxonomic groups, viz. Hyphomycetes, Meliolales, Schiffnerula and its anamorphic forms, Asterinales, Meliolinaceae, etc. Of these, the fungi belonging to Meliolales can be distinguished by their two celled appressoriate mycelium, setae, presence of globose perithecia with setae, appendages, etc. These are the unique group of fungi which are distinguished very easily. This work gives a clue to the identification of the meliolaceous fungi at a glance based on the hosts and their families. Meliolaceous fungi belong to the order Meliolales and which constitutes two families:

\section{The order Meliolales}

Meliolales Gaumann ex Hawksworth \& O. Eriksson, Systema Ascomycetum 5: 142, 1986.

Parasites on vascular plants. Mycelium mostly superficial, appressoriate. Appressoria mostly two celled, rarely many celled. Phialides unicellular. Ascomata flattened-globose to globose, \pm ostiolate, peridium smooth, surface cells protruded, often supplemented with setae, appendages; asci on basal hymenium, unitunicate, 2-8 spored, clavate to cylindrical, persistent or evanescent; ascospores 1-4 septate, brown at maturity.

Type family: Meliolaceae

\section{Discussion}

The order Meliolales represents two families to include eleven genera and 2300 species and infra specific taxa. India represents 613 taxa which forms $26.6 \%$ of the world's meliolaceous fungal flora. The monotypic genera, Pauhia and Endomeliola are not represented in India. In India, the genus Meliola scores highest number of species and infra specific taxa (453), followed by the genus Asteridiella (73), Irenopsis (31), Amazonia (28), Armatella (13), Appendiculella (10), the genera Basavamyces and Prataprajella (2) and the genus Ectendomeliola is a monotypic genus. Only the recently described genera, Basavamyces and Ectendomeliola are endemic to the Indian continent.

The reported number of flowering plants in India is ca.18000 species (the number varies from 16,500 to 19,395$)$ distributed among $c a .390$ families (the number varies from 247-315) (Mudgal \& Hajra 1997-99; Sharma et al. 1997; Karthikeyan 2000). Of the total known flowering plants, 766 species $(42.5 \%)$ found infected with these fungi belonging to 349 host genera (of the known 2900 genera, 12\%) distributed among 104 families $(31 \%)$.

Of the total numbers of flowering plant families in India, 104 are infected with meliolaceous fungi, Lauraceae represents highest number (49 taxa), Rubiaceae (40 taxa) followed by Fabaceae (39 taxa), Euphorbiaceae (32 taxa), Rutaceae (26 taxa), Apocynaceae (24 taxa), Myrtaceae and Sapindaceae (18 taxa each), Anacardiaceae and Oleaceae (17 taxa each), Meliaceae (16 taxa), Poaceae and Caesalpinaceae (12 taxa each), 
Table 1. Familes of plants, fungal genera, host plants and their associated fungal species

\begin{tabular}{|c|c|c|c|c|}
\hline SNo. & Host Family & Fungal Genera & Host & Fungal Species \\
\hline 1. & Acanthaceae 9 & Meliola & $\begin{array}{l}\text { Lepidagathis sp. } \\
\text { Diotacanthus sp. } \\
\text { Justicia sp. } \\
\text { Phaulopsis imbricata (Forssk.) Sweet } \\
\text { Strobilanthus sp. } \\
\text { Blepharis asperima Nees } \\
\text { Rungia sisparensis T. And. } \\
\text { Dicliptera foetida (Forssk.) Blatter } \\
\text { Barleria strigosa Willd. } \\
\text { Nilgirianthus heyneanus (Nees) Bremek } \\
\text { Strobilanthes heyeanus Nees } \\
\text { S. reticulates Stapf. } \\
\text { Strobilanthes rugosus Wight } \\
\text { Strobilanthes sp. } \\
\text { S. amabilis (Clarke) Bremek }\end{array}$ & $\begin{array}{l}\text { Asteridiella acanthacearum Hosag. } \\
\text { Asteridiella diotacanthi Hosag. et al. } \\
\text { Asteridiella justiciae Hosag. \& Rajkumar } \\
\text { Asteridiella phaulopsidis Hosag. } \\
\text { Asteridiella strobilanthicola Hosag. et al. } \\
\text { Meliola acanthacearum Hansf. var. occidentalis Hansf. } \\
\text { Meliola barleriae Srinivasulu } \\
\text { Meliola nilgirianthi Hosag. }\end{array}$ \\
\hline 2. & Agavaceae 1 & Meliola & Dracena terniflora Roxb. & Meliola dracenae-terniflorae Hosag. \\
\hline 3. & Anacardiaceae 17 & $\begin{array}{l}\text { Appendiculella } \\
\text { Asteridiella } \\
\text { Meliola }\end{array}$ & $\begin{array}{l}\text { Anacardium occidentale L. } \\
\text { Mangifera sp } \\
\text { Semecarpus anacardium L.f. } \\
\text { Anacrdium occidentale L. } \\
\text { Buchanania lanceolata Wight } \\
\text { B. lanzan Spreng. } \\
\text { uchaninia lanzan Spreng. } \\
\text { Lannea coromandelica (Houtt.) Merr. } \\
\text { Gluta travancorica L. } \\
\text { Holigarna ferruginea March. } \\
\text { H. grahamii (Wight) Kurz } \\
\text { H. ornottiana Hook.f } \\
\text { Lannea coromandelica (Houtt.) Merr. } \\
\text { Mangifera indica L. } \\
\text { Nothopegia colebrookiana (Wight) BI. } \\
\text { N. heyneana (Hook.f. Gamble) } \\
\text { N. racemosa (Dalz.) Ramam. } \\
\text { Semecarpus anacardium L.f. } \\
\text { Semecarpus travancorica Bedd. } \\
\text { Semecarpus sp. } \\
\text { Semecarpus travancorica Bedd. } \\
\text { Mackenziea gracilis (Bedd.) Bremek. }\end{array}$ & $\begin{array}{l}\text { Appendiculella anacardii Prameela \& Choudhry } \\
\text { Asteridiella mangiferae Jana et al. } \\
\text { Meliola abrahamii Hosag. et al. } \\
\text { Meliola anacardii Zimm. } \\
\text { Meliola ardigoosii Hosag. \& Abraham } \\
\text { Meliola buchananiicola Hosag. } \\
\text { Meliola geniculata Sydow \& Butler } \\
\text { Meliola glutae Hosag. \& Abraham } \\
\text { Meliola holigarnae Stev. } \\
\text { Meliola lanneae Hosag. \& Manoj. } \\
\text { Meliola mangiferae Earle } \\
\text { Meliola nothopegiae Hansf. } \\
\text { Meliola semecarpi-anacardii Hosag. } \\
\text { Meliola semecarpicola Hansf. } \\
\text { Meliola shivarajui Hosag. \& Kamar. } \\
\text { Meliola travancoricae Hosag. } \\
\text { Meliola mackenzieae Hosag. et al. }\end{array}$ \\
\hline 4. & Ancistrocladaceae 1 & Meliola & Ancistrocladus heyneanus Wall. ex Grah. & Meliola ancistrocladi Hosag. \\
\hline 5. & Angiopteridaceae 1 & Meliola & Angiopteris evecta (Forst.) Hoff. & Meliola angiopteridis Hansf. var. indica Hosag. \\
\hline 6. & Annonaceae 6 & $\begin{array}{l}\text { Amazonia } \\
\text { Irenopsis } \\
\text { Meliola }\end{array}$ & $\begin{array}{l}\text { Goniothalamus wightii Hook.f. \& Thoms. } \\
\text { G. wyanadensis (Bedd.) Bedd. } \\
\text { Goniothalamus rhynchantherus Dunn. } \\
\text { Artabotrys zeylanicus Hook.f. \& Thoms. } \\
\text { Mitrephora heyneana (Hook.f. \& Thoms.) Thw. } \\
\text { Meiogyne pannosa (Dalz.) Sinclair } \\
\text { (Unona pannosa Dalz.) } \\
\text { Meiogyne pannosa (Dalz.) Sinclair } \\
\text { (Unona pannosa Dalz.) }\end{array}$ & $\begin{array}{l}\text { Amazonia goniothalami Hosag. et al. } \\
\text { Irenopsis goniothalami Hosag. \& Abraham } \\
\text { Meliola artabotrydicola Hosag. \& Abraham } \\
\text { Meliola mitrephorae Hosag. \& Rajendran } \\
\text { Meliola unonae Hosag. \& Abraham } \\
\text { Meliola unonicola Hosag. \& Abraham }\end{array}$ \\
\hline 7. & Apocynaceae 24 & $\begin{array}{l}\text { Asteridiella } \\
\text { Meliola }\end{array}$ & $\begin{array}{l}\text { Wrightia coccinea Sims. } \\
\text { Alstonia scholaris (L.) R. Br. } \\
\text { Anodendron paniculatum (Roxb.) DC. } \\
\text { Carissa carandas L. } \\
\text { Carissa sp. } \\
\text { Carissa spinarum L. } \\
\text { Chilocarpus atrovirens (G. Don) Blume } \\
\text { Ellertonia rheedi Wight } \\
\text { Tabernaemontana heyneana Wall. } \\
\text { (Ervatamia heyneana T.Cooke) } \\
\text { Ichnocarpus frutescens (L.) R. Br. } \\
\text { Holarrhena sp. } \\
\text { H. pubescens (Buch-Ham.) Wallich ex G. Don } \\
\text { Holarrhena pubescens (Buch-Ham.) } \\
\text { Wallich ex G. Don } \\
\text { Hunteria zeylanica (Retz.) Gard. ex Thw. } \\
\text { (H. corymbosa Roxb. var. roxburgiana Trin.) } \\
\text { Ichnocarpus frutescens (L.) R. Br. } \\
\text { Ichnocarpus frutescense (L.) R. Br. } \\
\text { Ichnocarpus sp. } \\
\text { Carissa carandus L. } \\
\text { Tabernaemontana heyneana Wall. } \\
\text { Tabernaemontana sp. } \\
\text { Alstonia scholaris (L.) R. Br. }\end{array}$ & $\begin{array}{l}\text { Asteridiella wrightiae Jana et al. } \\
\text { Meliola alstoniae Koord. } \\
\text { Meliola anodendricola Hosag. } \\
\text { Meliola carissae Doidge var. indica Hansf. } \\
\text { Meliola carissae Doidge var. spinari Hosag. } \\
\text { Meliola chilocarpi Hosag. \& Abraham } \\
\text { Meliola ellertoniae Hosag. \& Abraham } \\
\text { Meliola ervatamiae Hosag. } \\
\text { Meliola frutescentis Hosag. et al. } \\
\text { Meliola holarrhenae Hansf. \& Thirum. } \\
\text { Meliola holarrhenae-pubescens Jana. et al. } \\
\text { Meliola hunteriae Hosag. } \\
\text { Meliola ichnocarpi Hansf. \& Thirum } \\
\text { Meliola ichnocarpi-volubili Hansf. } \\
\text { Meliola integripoda Hosag. et al. } \\
\text { Meliola pepparaensis Hosag. \& Abraham } \\
\text { Meliola peringamalaensis Hosag. \& Kamar. }\end{array}$ \\
\hline
\end{tabular}




\begin{tabular}{|c|c|c|c|c|}
\hline SNo. & Host Family & Fungal Genera & Host & Fungal Species \\
\hline & & & $\begin{array}{l}\text { Rauvolfia hookeri Srinivasan \& Chithra } \\
\text { Holarrhena antidysenterica } \\
\text { (Roxb. ex Fleming) Wall. } \\
\text { Plumeria alba L. } \\
\text { Tabernaemontana sp. } \\
\text { Wrightia tinctoria (Roxb.) R. Br. } \\
\text { Tabernaemontana sp. } \\
\text { Apocynaceae (indet.) }\end{array}$ & $\begin{array}{l}\text { Meliola rauvolfiae Mibey } \\
\text { Meliola simillima Ellis \& Everh. var.major Hansf. } \\
\text { Meliola srinivasului Hosag. } \\
\text { Meliola tabernaemontanae Speg. } \\
\text { Meliola tabernaemontanae Speg. var. wrightiae Hosag. et al. } \\
\text { Meliola tabernaemontanicola Hansf. \& Thirum. } \\
\text { Meliola thiruvananthapurica Hosag. \& Abraham }\end{array}$ \\
\hline 8. & Aquifoliaceae 2 & Meliola & $\begin{array}{l}\text { Ilex malabarica Beddome } \\
\text { Ilex sp. }\end{array}$ & $\begin{array}{l}\text { Meliola ilicis-malabaricae Hosag. \& Raghu } \\
\text { Meliola khasiensis Hansf. }\end{array}$ \\
\hline 9. & Araceae 1 & Asteridiella & Pothos scandens L. & Asteridiella pothodis (Hansf. \& Thirum.) Hansf. \\
\hline 10. & Araliaceae 3 & Meliola & $\begin{array}{l}\text { Brassaiopsis sp. } \\
\text { Schefflera venulosa (Wight \& Arn.) Harms } \\
\text { Hedera helix L. }\end{array}$ & $\begin{array}{l}\text { Meliola brassaiopsidis Hosag. } \\
\text { Meliola dichotoma Burk. \& Curt. var. kusanoi (Henn.) Hansf. } \\
\text { Meliola payakii Hosag. }\end{array}$ \\
\hline 11. & Arecaceae 2 & Meliola & $\begin{array}{l}\text { Phoenix sylvestris Roxb. } \\
\text { Phoenix sp. } \\
\text { Caryota urens L. }\end{array}$ & $\begin{array}{l}\text { Meliola palmicola Wint. var. africana Hansf. } \\
\text { Meliola caryotae Srinivasulu }\end{array}$ \\
\hline 12. & Asclepiadaceae 11 & Meliola & $\begin{array}{l}\text { Ceropegia sp. } \\
\text { Cynanchum sp } \\
\text { Tylophora tenuis BI. } \\
\text { Gymnema sylvestre (Retz.) R. Br. ex Schultes } \\
\text { Gymnema sp. } \\
\text { Asclepiadaceae (indet.) } \\
\\
\text { Tylophora tenuis BI. } \\
\text { Toxocarpus beddomei Gamble } \\
\text { Tylophora indica (Burm.f.) Merr. } \\
\text { Tylophora caparidifolia Wight \& Arn. }\end{array}$ & $\begin{array}{l}\text { Meliola ceropegiae Hosag. \& Ramachandran } \\
\text { Meliola cynanchi Hosag. et al. } \\
\text { Meliola dimorphochaeta Patil \& Mahamul. } \\
\text { Meliola gymnemae Jana. et al. } \\
\text { Meliola telosmae Rehm var. } \\
\text { radhanagariensis Hosag. } \\
\text { Meliola telosmae Rehm. var. bogoriensis Hansf. } \\
\text { Meliola telosmae Rehm var. indica Hosag. et al. } \\
\text { Meliola toxocarpi Hosag. \& Antony } \\
\text { Meliola tylophorae Hosag. \& Manoj. } \\
\text { Meliola tylophorae- indicae Hosag. }\end{array}$ \\
\hline 13. & Asteraceae 3 & Meliola & $\begin{array}{l}\text { Vernonia arborea Buch.-Ham. } \\
\text { (V. monosis Clarke) } \\
\text { Vernonia arborea Buch.-Ham. } \\
\text { (V. monosis Benth. ex Clarke) } \\
\text { Coereopsis auristosa Michx. }\end{array}$ & $\begin{array}{l}\text { Asteridiella cyclopoda (Stev.) Hansf. } \\
\text { Asteridiella cyclopoda (Stev.) Hansf. var. vernoniae } \\
\text { Hosag. et al. } \\
\text { Meliola coreopsidis Thite \& Kulkarni }\end{array}$ \\
\hline 14. & Bignoniaceae 2 & $\begin{array}{l}\text { Asteridiella } \\
\text { Meliola }\end{array}$ & $\begin{array}{l}\text { Stereospermum colais (Buch. - Ham. } \\
\text { ex Dillwyn.) Mabb. } \\
\text { Heterophragma roxburghii DC. } \\
\text { Pagenelia longifolia (Willd.) K.Schum. } \\
\text { (P. rheedii DC.) }\end{array}$ & $\begin{array}{l}\text { Asteridiella schlegeliae (Stev.) Hansf. var. stereospermi } \\
\text { Hosag. \& Raghu } \\
\text { Meliola crescentiae Stev. }\end{array}$ \\
\hline 15. & Boraginaceae 2 & $\begin{array}{l}\text { Asteridiella } \\
\text { Meliola }\end{array}$ & $\begin{array}{l}\text { Ehretia canarensis (Clarke) Gamble } \\
\text { Ehretia canarensis (Clarke) Gamble }\end{array}$ & $\begin{array}{l}\text { Asteridiella ehretiae Hosag. \& Raghu } \\
\text { Meliola ehretiicola Hosag. }\end{array}$ \\
\hline 16. & Burseraceae 1 & & Carnarium strictum Roxb. & Meliola canarii Sydow \\
\hline 17. & Caesalpiniaceae 12 & Meliola & $\begin{array}{l}\text { Cassia fistula L. } \\
\text { Cassia fistula L. } \\
\text { Cassia sp. } \\
\text { Moullava spicata (Dalz.) Nicolson } \\
\text { Bauhinia phoenicea Wight \& Arn. } \\
\text { Bauhinia racemosa Lam. } \\
\text { Caesalpinia sappan L. } \\
\text { Cassia fistula I. } \\
\text { Kingiodendron pinnatum (Roxb.) Harms } \\
\text { Bauhinia sp. } \\
\text { Cassia surattensis Burm. } \\
\text { Tamarindus indica L. }\end{array}$ & $\begin{array}{l}\text { Meliola aethiops Sacc. } \\
\text { Meliola aethiops Sacc. var. cassiae Rao } \\
\text { Meliola aethiops Sacc. var. keralica Hosag. } \\
\text { Meliola aethiops Sacc. var. moullavae Hosag. \& Raghu } \\
\text { Meliola bauhiniae-phoeniceae Hosag. et al. } \\
\text { Meliola bauhiniicola Yamam. } \\
\text { Meliola caesalpiniae Hansf. \& Deight. var. indica Hosag. } \\
\text { \& H. Biju } \\
\text { Meliola cassiae-fistulae Hosag. \& Manoj. } \\
\text { Meliola kingiodendri Hosag. et al. } \\
\text { Meliola kulathupuzhaensis Hosag. \& S. Justin P. Jacob } \\
\text { Meliola surattensis Hosag. et al. } \\
\text { Meliola tamarindi Sydow \& Sydow }\end{array}$ \\
\hline 18. & Capparaceae 2 & $\begin{array}{l}\text { Asteridiella } \\
\text { Meliola }\end{array}$ & $\begin{array}{l}\text { Capparaceae (indet.) } \\
\text { Capparis divaricata Lam. }\end{array}$ & $\begin{array}{l}\text { Asteridiella capparidigena Hosag. } \\
\text { Meliola capparidicola Hosag. }\end{array}$ \\
\hline 19. & Caprifoliaceae 3 & Meliola & $\begin{array}{l}\text { Viburnum punctatum Buch.-Ham. ex D. Don } \\
\text { Leycesteria glaucophylla Hook.f. }\end{array}$ & $\begin{array}{l}\text { Meliola aequatoriensis Petrak } \\
\text { Meliola goosii Hosag. } \\
\text { Meliola leycesteriae Kar \& Maity }\end{array}$ \\
\hline 21 & Celastraceae 8 & $\begin{array}{l}\text { Appendiculella } \\
\text { Asteridiella } \\
\text { Meliola }\end{array}$ & $\begin{array}{l}\text { Maytenus rothiana (Laws.) Lobreu-Callen } \\
\text { Maytenus emerginata (Willd.) Ding Hu } \\
\text { (Gymnosporia montana (Roth) Benth.) } \\
\text { Celastrus paniculatus Willd. } \\
\text { Lophopetalum wightianum Arn. } \\
\text { Bhesa indica (Bedd.) Ding Hou } \\
\text { Lophopetalum sp. } \\
\text { Pleurostylia sp. } \\
\text { Euonymus indicus Heyne ex Roxb. }\end{array}$ & $\begin{array}{l}\text { Amazonia mayteni Hosag. } \\
\text { Amazonia patilii Hosag. } \\
\text { Appendiculella gloriosa (Doidge) Hansf. } \\
\text { Asteridiella lophopetali Hosag. \& Raghu } \\
\text { Meliola bhesae Hosag. } \\
\text { Meliola chennaiana Hosag. \& Goos } \\
\text { Meliola celastracearum Hosag. \& B.R. Dayal } \\
\text { Meliola euonymicola Hosag. }\end{array}$ \\
\hline
\end{tabular}

22. Clusiaceae 3 Appendiculella Calophyllum austroindicum Kosterm.

Appendiculella calophylli (Stev.) Toro var. apetali Hosag. et al. C. apetalum Willd. 


\begin{tabular}{|c|c|c|c|c|}
\hline SNo. & Host Family & Fungal Genera & Host & Fungal Species \\
\hline & & Meliola & $\begin{array}{l}\text { Garcinia spicata (Wight \& Arn.) Hook.f. } \\
\text { Mammia suriga (Buch.-Ham. ex Roxb.) } \\
\text { Kosterm. (Ochrocarpus longifolius } \\
\text { (Wight) Benth. ex T. And.) }\end{array}$ & $\begin{array}{l}\text { Meliola garciniae Yates. } \\
\text { Meliola ochrocarpi Thite \& Patil }\end{array}$ \\
\hline 23. & Combretaceae 2 & $\begin{array}{l}\text { Amazonia } \\
\text { Asteridiella }\end{array}$ & $\begin{array}{l}\text { Combretum decandrum Roxb. } \\
\text { Terminalia chebula Retz. } \\
\text { T. paniculata Roth }\end{array}$ & $\begin{array}{l}\text { Amazonia henryi Hosag. } \\
\text { Asteridiella combreti (Stev.) Hansf. var. leonensis Hansf. }\end{array}$ \\
\hline 24. & Connaraceae 4 & Meliola & $\begin{array}{l}\text { Connarus monocarpus L. } \\
\text { Rourea praineana Talbot } \\
\text { Connarus sclerocarpus (Wight \& Arn.) Schell. } \\
\text { Connarus wightii Hook.f. }\end{array}$ & $\begin{array}{l}\text { Meliola agumbensis (Subhedar \& Rao) Hosag. } \\
\text { Meliola connari Yates } \\
\text { Meliola connari Yates var. indica Hosag. } \\
\text { Meliola roureae Sydow var. major Hansf. \& Deight. }\end{array}$ \\
\hline 25. & Convolvulaceae 5 & Meliola & $\begin{array}{l}\text { Argyreia hirsute Arn. } \\
\text { A. populifolia Choisy } \\
\text { Ipomoea sp. } \\
\text { Erycibe paniculata Roxb. } \\
\text { Porona paniculata Roxb. } \\
\text { Argyereia sp. } \\
\text { Argyereia elliptica Choisy } \\
\text { A. hookeri Clarke } \\
\text { A. setosa (Roxb.) Choisy } \\
\text { Merremia umbellata (L.) Hall.f. } \\
\text { Argyereia elliptica (Roth) Choisy } \\
\text { A. hookeri Clarke } \\
\text { A. speciosa Sweet }\end{array}$ & $\begin{array}{l}\text { Meliola clavulata Wint. } \\
\text { Meliola erycibes-paniculatae Hosag. } \\
\text { Meliola malacotricha Speg. } \\
\text { Meliola malacotricha Speg. var. major Beeli }\end{array}$ \\
\hline 26. & Cornaceae 1 & Asteridiella & Mastixia arborea (Wight) Bedd. & Asteridiella mastixiae Hosag.\&. Raghu \\
\hline 27. & Cucurbitaceae 3 & Asteridiella & $\begin{array}{l}\text { Trichosanthes tricuspidata Lour. } \\
\text { (T. palmata Roxb.) } \\
\text { Citrulla sp. } \\
\text { Mukia sp. } \\
\text { Momordica sp. }\end{array}$ & $\begin{array}{l}\text { Asteridiella confragosa (Sydow \& Sydow) Hansf. } \\
\text { Asteridiella triloba (Wint.) Hansf. } \\
\text { Asteridiella triloba (Wint.) Hansf. var. momordicae Hosag. et al. }\end{array}$ \\
\hline 28. & Cyperaceae 1 & Meliola & Rhynchospora corymbosa (L.) Britt. & Meliola tibigirica Hosag. \& Abraham \\
\hline 29. & Daphniphyllaceae 1 & Amazonia & Daphniphyllum neilgherrense (Wight) Rosenth. & $\begin{array}{l}\text { Amazonia daphniphylli } \\
\text { M.S. Patil }\end{array}$ \\
\hline 30. & Dichapetalaceae 1 & Meliola & Dichapetalum gelonioidea (Roxb.) Engl. & Meliola dichapetali Hansf. \& Thirum. \\
\hline 31. & Dilleniaceae 1 & Asteridiella & Dillenia pentagyna Roxb. & Asteridiella dilleniae Hosag. \& Kamarudeen \\
\hline 32. & Ebenaceae 6 & $\begin{array}{l}\text { Asteridiella } \\
\text { Meliola }\end{array}$ & $\begin{array}{l}\text { Diospyros candolleana Wight } \\
\text { Diospyros sp. } \\
\text { Diospyros montana Roxb. } \\
\text { D. nigrescens (Dalzel) Saldhana } \\
\text { D. pruriens Dalz. } \\
\text { D. sylvatica Roxb. } \\
\text { D. malabarica (Desr.) Kostel. } \\
\text { Diospyros sp. } \\
\text { Diospyros nigrescens (Dalzel) Saldhana } \\
\text { (Maba nigriscens Dalzel) } \\
\text { Diospyros sp. } \\
\text { Diospyros buxifolia (Blume) Hiern. } \\
\text { (D. microphylla Bedd.) }\end{array}$ & $\begin{array}{l}\text { Meliola diospyri Sydow \& Sydow var. yatesiana (Trott.) } \\
\text { Hansf. \& Deight. } \\
\text { Meliola diospyricola Hansf. } \\
\text { Meliola megalocarpa Sydow var. microspora Hosag. }\end{array}$ \\
\hline 33. & Elaegnaceae 1 & Meliola & $\begin{array}{l}\text { Elaegnus kologa Schlecht (E. latifolia L.) } \\
\text { E. indica Serv. }\end{array}$ & Meliola elaegni Hansf. \& Thirum. \\
\hline 34. & Elaeocarpaceae 1 & Asteridiella & Elaeocarpus tuberculatus Roxb. & $\begin{array}{l}\text { Asteridiella } \\
\text { elaeocarpi-tuberculati Hosag. }\end{array}$ \\
\hline 35. & Ericaceae 4 & $\begin{array}{l}\text { Amazonia } \\
\text { Appendiculella } \\
\text { Asteridiella }\end{array}$ & $\begin{array}{l}\text { Agapetes sp. } \\
\text { Gaultheria fragrantissima Wall. } \\
\text { Gaultheria indica Wall. } \\
\text { Pentapterygium serpens Klotsch }\end{array}$ & $\begin{array}{l}\text { Amazonia karii Hosag. \& Balakr. } \\
\text { Appendiculella gaultheriae Hosag. } \\
\text { Asteridiella gaultheriae Hosag. et al. } \\
\text { Asteridiella pentapterygii Kar \& Maity }\end{array}$ \\
\hline 36. & Erythropalaceae 1 & Meliola & Erythropalum populifolium (Arn.) Mast. & Meliola erythropali Hosag. \\
\hline 37. & Erythroxylaceae 1 & Meliola & Erythroxylum obtusifolium (Wight) Hook.f. & Meliola erythroxylifolii Batista \& Vital \\
\hline 38. & Euphorbiaceae 32 & Asteridiella & $\begin{array}{l}\text { Croton malabaricus Muell.-Arg. } \\
\text { C. zeylanicus Muell.-Arg. } \\
\text { Croton zeylanicus Muell.-Arg. } \\
\text { (C. reticulates Heyne ex Muell.-Arg.) } \\
\text { Macaranga peltata (Roxb.) Muell.- Arg. } \\
\text { Glochidion sp. } \\
\text { Mallotus philippensis (Lam.) Muell.-Arg. } \\
\text { Macaranga peltata Muell.-Arg. } \\
\text { Mallotus alba Muell.-Arg. } \\
\text { M. philippensis (Lam.) Muell.-Arg. }\end{array}$ & $\begin{array}{l}\text { Asteridiella crotonicola Hosag. \& Abraham } \\
\text { Asteridiella crotonis Hosag. } \\
\text { Asteridiella entebbeensis (Hansf. \& Stev.) Hansf. } \\
\text { Asteridiella entebbeensis (Hansf. \& Stev.) Hansf. var. } \\
\text { glochidii Hosag. et al. } \\
\text { Asteridiella kombeensis Hosag. } \\
\text { Asteridiella macarangicola Hosag. } \\
\text { Asteridiella malloti (Hansf. \& Thirum.) Hansf. }\end{array}$ \\
\hline
\end{tabular}




\begin{tabular}{|c|c|c|c|c|}
\hline SNo. & Host Family & Fungal Genera & Host & Fungal Species \\
\hline & & Meliola & $\begin{array}{l}\text { M. tetracoccus (Roxb.) Kurz (M. albus Muel } \\
\text { Mallotus philippensis (Lam.) Muell.- Arg. } \\
\text { Madhuca longifolia (L.) Macbr. var. } \\
\text { latifolia (Roxb) A. Chev. } \\
\text { Mallotus sp } \\
\text { Mallotus repandus (Willd.) Muell.-Arg. } \\
\text { Mallotus roxburghianus Muell. } \\
\text { Croton zeylanicus Muell.-Arg. } \\
\text { Croton sp. } \\
\text { Actephila excelsa (Dalz.) Muell.-Arg. } \\
\text { Agrostistachys borneensis Becc. } \\
\text { (A.meeboldii Pax \& Hoffm.) } \\
\text { Excoecaria crenulata Wight } \\
\text { Croton malabaricus Bedd. } \\
\text { Drypetes macrophylla (BI.) Pax \& Hoffm. } \\
\text { Epiprinus mallotiformis (Muell.-Arg.) Croizat } \\
\text { Farenheitia zeylanica (Thw.) Airyshaw } \\
\text { Glochidion velutinum Wight } \\
\text { Glochidion ellipticum Wight } \\
\text { G. hohenackeri Bedd. } \\
\text { Bridelia sp. } \\
\text { Jatropha glandulifera Roxb. } \\
\text { Glochidion sp. } \\
\text { Mallotus philippensis (Lam.) Muell.-Arg. } \\
\text { Ostodes paniculata Blume } \\
\text { Euphorbiaceae (indet.) } \\
\text { Homonoia riparia Lour. } \\
\text { Glochidion sp. } \\
\text { Trewia nodiflora L } \\
\text { T. polycarpa Benth. ex Hook. } \\
\text { Antidesma zeylanica Laun. (A. alexiteria L.) }\end{array}$ & $\begin{array}{l}\text { All.-Arg.) } \\
\text { Asteridiella malloticola (Yamam.) Hansf. } \\
\text { Asteridiella wyanadensis Hosag. et al. } \\
\text { Irenopsis crotonis (Stev. \& Tehon) Stev. } \\
\text { Irenopsis hypophyllae Jana. et al. } \\
\text { Irenopsis paulensis Hansf. } \\
\text { Meliola actephilae Hosag. et al. } \\
\text { Meliola agrostistachydis Hosag. \& Rajkumar } \\
\text { Meliola chandleri Hansf. } \\
\text { Meliola crotonis. } \\
\text { malabarici Hosag. } \\
\text { Meliola drypeticola Hosag. } \\
\text { Meliola farenheitiae Hosag. \& Abraham } \\
\text { Meliola glochidii Stev. \& Rold. ex Hansf. var. velutini Hosag. } \\
\text { Meliola glochidiicola Yamam. } \\
\text { Meliola himalayensis Kapoor } \\
\text { Meliola jatrophae Stev. } \\
\text { Meliola karnatakensis Hosag. et al. } \\
\text { Meliola malloticola Hosag. } \\
\text { Meliola ostodis Kapoor } \\
\text { Meliola radhanagariensis Hosag. } \\
\text { Meliola ramosii Sydow \& Sydow } \\
\text { Meliola thiteana Hosag. } \\
\text { Meliola trewiae Hosag. } \\
\text { Meliola vivekananthanii Hosag. et al. }\end{array}$ \\
\hline 39. & Fabaceae 39 & $\begin{array}{l}\text { Asteridiella } \\
\text { Meliola }\end{array}$ & $\begin{array}{l}\text { Millettia rubiginosa Wight \& Arn. } \\
\text { Atylosia lineata Wight \& Arn. } \\
\text { Puereria tuberosa DC. } \\
\text { Puereria sp. } \\
\text { Pueraria tuberosa DC. } \\
\text { Desmodium gyrans DC. } \\
\text { Millettia rubiginosa Wight \& Arn. } \\
\text { Millettia rubiginosa Wight \& Arn. } \\
\text { Desmodium telifolia } \\
\text { D. triquetrum (L.) DC } \\
\text { D. velutinum (Willd.) DC. } \\
\text { Dolichos trilobus L. (D. fulcatus Kein ex Willd } \\
\text { Butea monosperma (Lam.) Taub. } \\
\text { (B. frondosa Roxb.) } \\
\text { B. parviflora Roxb. } \\
\text { Calopogonium mucunoides Desv. } \\
\text { Clitoria ternatea L. } \\
\text { Desmodium laxiflorum DC. } \\
\text { Desmodium motorium (Houtt.) Merr. } \\
\text { Desmodium pulchellum (L.) Benth. } \\
\text { Desmodium triangulare (Retz.) Merr. } \\
\text { Desmodium triquetrum (L.) DC. } \\
\text { Desmodium velutinum (Willd.) DC. } \\
\text { Dolichus trilobus L. } \\
\text { D. trifolius } \\
\text { Erythrina variegata L. (E. indica Lam.) } \\
\text { E. stricta Roxb. } \\
\text { Flemingia semialata } \\
\text { Flemingia sp. } \\
\text { Ormosia travancorica Bedd. } \\
\text { Geissaspis cristata Wight \& Arn. } \\
\text { Gliricidia sepium (Jacq.) Walp } \\
\text { Millettia splendens Wight \& Arn. } \\
\text { Millettia racemosa (Wight \& Arn.) Benth. } \\
\text { Mucuna imbricata DC. } \\
\text { Mucuna hirsuta Wight \& Arn. } \\
\text { Mucuna pruriens (L.) DC. } \\
\text { Millettia pequensis Ali } \\
\text { Vigna khandalensis (Sant.) Raghu \& } \\
\text { Wadhwa (Phaseolus khandalensis Sant.) } \\
\text { Pongamia pinnata (L.) Pierre } \\
\text { Pseudarthria viscida (L.) Wight \& Arn. } \\
\text { Pterocarpus marsupium Roxb. } \\
\text { Pycnospora sp. } \\
\text { Spatholobus parviflorus (Roxb. ex DC.) Kuntze } \\
\text { S. roxburghii Benth. } \\
\text { Spatholobus sp. } \\
\text { De _ }\end{array}$ & 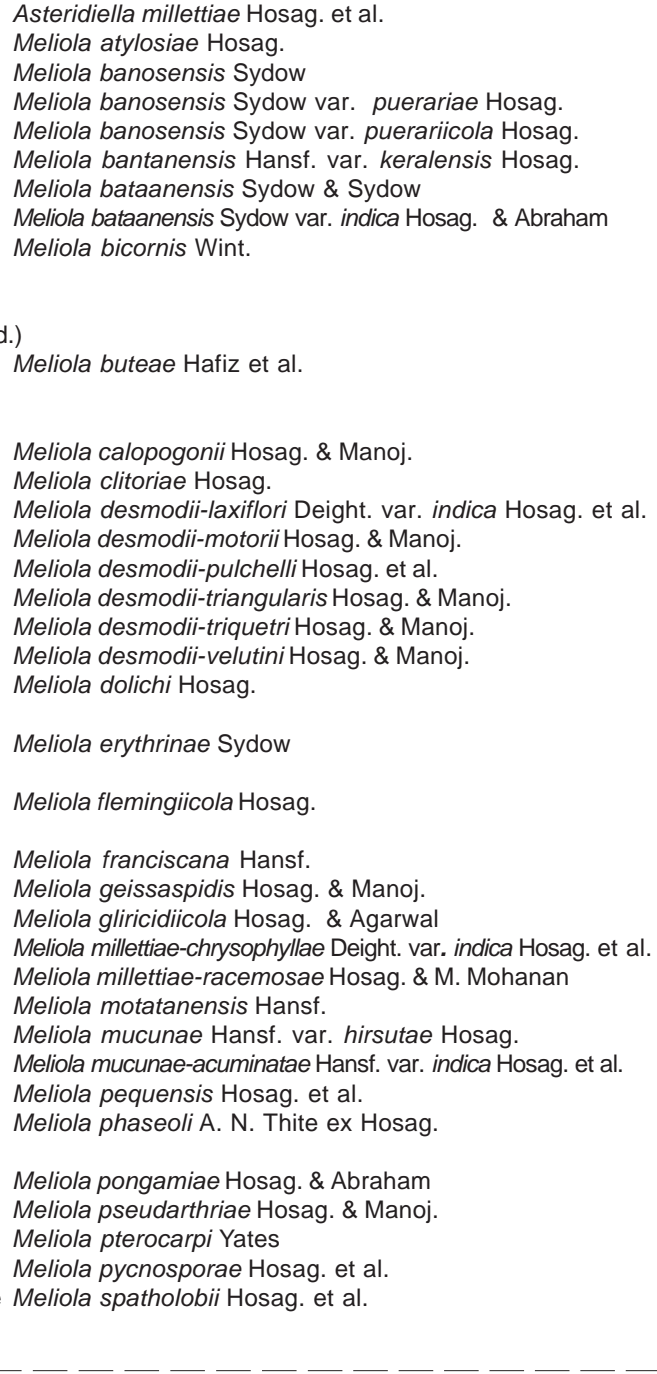 \\
\hline
\end{tabular}




\begin{tabular}{lllll}
\hline SNo. & Host Family & Fungal Genera & Host & Fungal Species
\end{tabular}

\begin{tabular}{|c|c|c|}
\hline 40. & Fagaceae 6 & $\begin{array}{l}\text { Amazonia } \\
\text { Asteridiella } \\
\text { Meliola }\end{array}$ \\
\hline 41. & Flacourtiaceae 5 & $\begin{array}{l}\text { Amazonia } \\
\text { Asteridiella } \\
\text { Meliola }\end{array}$ \\
\hline 42. & Gentianaceae 1 & Meliola \\
\hline 43. & Gesneriaceae 1 & Asteridiella \\
\hline 44. & Gnetaceae 1 & Meliola \\
\hline 45. & Hemmamalidaceae 1 & Meliola \\
\hline 46. & Hippocrateaceae 2 & Meliola \\
\hline 47. & Icacinaceae 7 & $\begin{array}{l}\text { Amazonia } \\
\text { Meliola }\end{array}$ \\
\hline
\end{tabular}

48.

Lamiaceae 4

Asteridiella

49.

Lauraceae $49 \quad$ Amazonia

Armatella
Millettia rubiginosa Wight \& Arn. Teramnus labialis (L.f.) Spreng. Uraria rufescens (DC.) Schindl.

Castenopsis armata Spanch

Quercus sp.

Quercus leucotrichophora A. Camus

Castonopsis hystrix A. DC.

Castenopsis armata Spanch

Quercus spicata Sm.

Flacourtia sp.

Casearia esculenta Roxb.

Scolopia crenata (Wight \& Arn.) Clos

Hydnocarpus laurifolius

$H$. pentandra (Buch-Ham) Oken

Scolopia crenata (Wight \& Arn.) Clos

Exacum tetragonum Roxb. (E.bicolor)

Didymocarpus humboldtianus Gard.

Gnetum ula Brogn.

Symingtonia populnea (R. Br.) Van Steenis Meliola symingtoniae Kapoor

Loecneriella obtusifolia (Roxb.) A.C. Smith Meliola oligomera Sydow Salacia sp.

Gomphandra coriacea Wight

Apodytes dimidiata E. Meyer ex Arn.

Notopodytes nimmoniana (Graham) Mabberly

Apodytes dimidiata E. Meyer ex Arn.

Gomphandra coriacea Wight

Sarcostigma kleini Wight

Sarcostigma kleinii Wight \& Arn.

Stemonurus tetrandrus Wall. ex Roxb.

Leucas sp.

Colebrookia oppositifolia Sm.

Hyptis suaveilense (L.) Poit.

H. capitata Jacq.

Pogostemon pubescens Benth.

Actionodaphne malabarica Balakr.

(A. hookeri sensu Bedd.)

Cinnamomum riparium Gamble

Actinodaphne sp.

Apollonias arnottii Nees

Cinnamomum malabatrum (Burm.f.) Blume

Litsea sp.

Cinnamomum zeylanicum $\mathrm{L}$.

C. macrocarpum Hook.f.

Cinnamomumsp.

Cinnamomum macrocarpum Hook.f.

C. malabatrum (Burm.f.) Blume

Cinnamomumsp.

Cryptocarya bourdillonii Gamble

Cryptocarya sp

Litsea coriacea (Heyne ex Meissner) Hook.f.

L. stocksii Hook.f.

L. wightii ana sensu Hook.f.

L. glabra (Wallich ex Nees) Hook.f.

L. glabrata (Wallich ex Nees) Hook.f.

L. deccanensis Gamble

Litsea sp.

Cinnamomum malabatrum (Burm.f.) Blume

C. sulphuratum

Cinnamomumsp.

Apollonias arnottii Nees

Litsea stocksii Hook.f

Persea macrantha (Nees) Kosteerm.

(Machilus macrantha Nees)

Litsea sp.

Persea sp.

Daphnedium pulcherrima

Neolitsea fischeri Gamble

N. scrobiculata (Meisner) Gamble

N. zeylanica Merr.

Neolitsea scrobiculata (Meisner) Gamble

Neolitsea sp.

Actinodaphne sp.

Meliola melanochaeta Sydow
Meliola salaciae Hansf.

Amazonia gomphandrae Hosag.

Meliola teramni Sydow var. millettiae Hosag.

Meliola teramnicola Hosag.

Meliola urariae Hosag.

Amazonia balakrishnanii Hosag.

Asteridiella quercina Hansf

Meliola bosei Hosag.

Meliola hystricis Kar \& Maity

Meliola mannii Hosag.

Amazonia flacourtiae Hosag. et al.

Asteridiella caseariicola Hosag.

Asteridiella scolopiae Hosag.

Meliola hydnocarpi Hansf. var. indica Hosag. \& Kamar.

Meliola scolopiae Doidge var. indica Hosag.

Meliola exaci Hosag.

Asteridiella cyrtandrae (Stev.) Hansf. var. didymocarpi Hosag.

Meliola gneti Hansf

Meliola chandrasekharanii Hosag.

Meliola dimidiatae Hosag.

Meliola gomphandrae Hosag. \& Abraham

Meliola sarcostigmaticola Hosag. et al.

Meliola sarcostigmatis Hosag.

Meliola stemonuri Hosag.

Asteridiella anastomosans (Wint.) Hansf.

Asteridiella colebrookiae Jana et al.

Meliola hyptidis Sydow

Meliola pogostemonis Hansf.

Amazonia actinodaphnes Hosag

Amazonia cinnamomi Hosag

Armatella actinodaphnes Hosag. et al.

Armatella apolloniadis Hosag. et al.

Armatella balakrishnanii Hosag.

Armatella caulicola Hosag. et al.

Armatella cinnamomi Hansf. \& Thirum.

Armatella cinnamomicola Hansf.

Armatella cryptocaryae Hosag.

Armatella indica Hosag.

Armatella kakachiana Hosag. \& Goos Armatella katumotoi Hosag.

Armatella litseae (P. Henn.) Theiss. \& Sydow

Armatella litseae (Henn.) Theiss. \& Sydow var. boninensis Katumoto \& Harada

Armatella phoebecola Hosag. 


\begin{tabular}{|c|c|c|c|c|}
\hline SNo. & Host Family & Fungal Genera & Host & Fungal Species \\
\hline & & $\begin{array}{l}\text { Basavamyces } \\
\text { Meliola }\end{array}$ & $\begin{array}{l}\text { Litsea sp. } \\
\text { Phoebe lanceolata Nees. } \\
\text { Litsea sp. } \\
\text { Litsea sp. } \\
\text { Actinodaphne malabarica Balakr. } \\
\text { Actinodaphne sp. } \\
\text { Beilschmiedia wightii (Nees) Benth. ex Hook.f. } \\
\text { Cinnamomum malabatrum (Burm.f.) Blume } \\
\text { Lauraceae (indet.) } \\
\text { Cinnamomum sp. } \\
\text { Cryptocarya bourdillonii Gamble } \\
\text { Litsea insignis Gamble } \\
\text { Persea macrantha (Nees) Kosterm. } \\
\text { Persea macrantha (Nees) Kosterm. } \\
\text { Actinodaphne sp. } \\
\text { Cryptocarya beddomei Gamble } \\
\text { Cinnamomum sp. } \\
\text { Actinodaphne hookeri Meissn. } \\
\text { Actinodaphne malabarica Balakr. } \\
\text { Litsea floribunda (Blume) Gamble } \\
\text { Litsea insignis Gamble } \\
\text { Litsea stocksii (Meisner) Hook.f.var. } \\
\text { glabrescense (Meisner) Hook.f. } \\
\text { Litsea floribunda (Bl.) Gamble } \\
\text { Litsea coriacea (Heyne ex Meisner) Hook.f. } \\
\text { Persea macrantha (Nees) Kosteerm. } \\
\text { (Machilus macrantha Nees) } \\
\text { Litsea angustima Litsea sp. } \\
\text { Neolitsea scrobiculata (Meisner) Gamble } \\
\text { (N. zeylanica Merr.) } \\
\text { Cryptocarya bourdillonii Gamble } \\
\text { (C. wightiana sensu Hook.f.) } \\
\text { Litsea sp. } \\
\text { Cryptocarya bourdillonii Gamble } \\
\text { Litsea sp. } \\
\text { Persea macrantha (Nees) Kosterm. } \\
\text { Litsea sp. } \\
\text { Actinodaphne sp. } \\
\text { Litsea sp. } \\
\text { Neolitsea sp. }\end{array}$ & $\begin{array}{l}\text { Basavamyces litseae Hosag. et al. } \\
\text { Basavamyces patilii Hosag. } \\
\text { Meliola actinodaphnecola Hosag. \& Abraham } \\
\text { Meliola banasuranii Hosag. et al. } \\
\text { Meliola beilschmediicola Hosag. } \\
\text { Meliola beilschmiediae Yamam. var. cinnamomicola Hosag. } \\
\text { Meliola cholakadensis Hosag. et al. } \\
\text { Meliola cinnamomi Hosag. \& Abraham } \\
\text { Meliola cryptocariicola Hosag. \& Raghu } \\
\text { Meliola drepanochaeta Sydow var. insignis Hosag. } \\
\text { Meliola floridensis Hansf. } \\
\text { Meliola floridensis Hansf. var. pudukadensis Hosag. } \\
\text { Meliola gooseana Hosag. \& Abraham } \\
\text { Meliola kakachiana Hosag. } \\
\text { Meliola kaveriappai Hosag. et al. } \\
\text { Meliola linderae Yamam. } \\
\text { Meliola linderae Yamam. var. microspora Hosag. \& Abraham } \\
\text { Meliola litseae Sydow\& Sydow var. floribundae Hosag. } \\
\text { Meliola litseae Sydow \& Sydow var. insignis Hosag. } \\
\text { Meliola litsea Sydow var. keralensis Hosag. } \\
\text { Meliola litseae Sydow \& Sydow var. microspora Hosag. } \\
\text { Meliola litseae Sydow \& Sydow var. rotundipoda Hansf. } \\
\text { Meliola machili Yamam. } \\
\text { Meliola mannavenansis Hosag. et al. } \\
\text { Meliola neolitseae Yamam. } \\
\text { Meliola patileana Hosag. } \\
\text { Meliola pudukadensis Hosag. } \\
\text { Meliola pushpangadanii Hosag. \& Abraham } \\
\text { Meliola ramacharii Hosag. } \\
\text { Meliola sempeiensis Yamam. var. nicobarica Lakshmanan et al. } \\
\text { Meliola shettyi Hosag. et al. } \\
\text { Meliola tetradeniae (Berk.) Theiss. \& Sydow }\end{array}$ \\
\hline 50. & Lecythidaceae 4 & Meliola & $\begin{array}{l}\text { Careya arborea Roxb. } \\
\text { Careya arborea Roxb. } \\
\text { Barringtonia acutangula Gaertn } \\
\text { Careya arborea Roxb. }\end{array}$ & $\begin{array}{l}\text { Meliola careyae (Stev.) Hosag. } \\
\text { Meliola careyae (Stev.) Hosag. var. indica Hosag. } \\
\text { Meliola indica Sydow } \\
\text { Meliola indica Sydow var. careyae Stev. }\end{array}$ \\
\hline 51. & Leeaceae 4 & $\begin{array}{l}\text { Amazonia } \\
\text { Irenopsis } \\
\text { Meliola }\end{array}$ & $\begin{array}{l}\text { Leea indica (Burm.f.) Merr. } \\
\text { L. macrophylla Roxb. } \\
\text { Leea indica (Burm.f.) Merr. } \\
\text { Leea indica (Burm.f.) Merr. } \\
\text { Leea asiatica (L.) Ridsdale } \\
\text { Leea sp. }\end{array}$ & $\begin{array}{l}\text { Amazonia leeae Hansf. \& Thirum. } \\
\text { Irenopsis leeae Hansf. var. indica Hosag. } \\
\text { Irenopsis leeae Hansf. var. javensis Hansf. } \\
\text { Meliola maredumilliana Hosag. \& M. Mohanan }\end{array}$ \\
\hline 52. & Lobeliaceae 1 & Meliola & Lobelia nicotianifolia Roth ex Schultes & Meliola lobeliae Stev. \\
\hline 53. & Loganiaceae 4 & Meliola & $\begin{array}{l}\text { Gardneria sp. } \\
\text { Gardneria ovata Wall. } \\
\text { Strychnos nux-vomica L. } \\
\text { Strychnos nux-vomica L. }\end{array}$ & $\begin{array}{l}\text { Meliola gardneriae Hansf. \& Thirum. } \\
\text { Meliola gardneriae Hansf. \& Thirum. var. indica Hosag. et al. } \\
\text { Meliola petchii Hansf. } \\
\text { Meliola spigeliae Hansf. }\end{array}$ \\
\hline 54. & Loranthaceae 3 & Meliola & $\begin{array}{l}\text { Dendropthoe falcata (L.f.) Etting } \\
\text { Helixanthera sp. } \\
\text { Loranthus sp. } \\
\text { Dendropthoe falcata (L.f.) Etting } \\
\text { Dendropthoe falcata (L.f.) Etting }\end{array}$ & $\begin{array}{l}\text { Meliola dendropthoeicola Hosag. \& Abraham } \\
\text { Meliola loranthacearum Hosag. \& Abraham } \\
\text { Meliola prataprajii Hosag. \& Abraham }\end{array}$ \\
\hline 55. & Lythraceae 1 & Meliola & Woodfordia fruticosa Kurz. & Meliola woodfordiae Srinivausulu \\
\hline 56. & Magnoliaceae 1 & Asteridiella & Michelia velutina DC. & Asteridiella micheliae Jana et al. \\
\hline 57. & Malvaceae 8 & $\begin{array}{l}\text { Amazonia } \\
\text { Asteridiella } \\
\text { Irenopsis }\end{array}$ & $\begin{array}{l}\text { Abutilon ramosum (Cav.) Guill. \& Perr. } \\
\text { Julostylis polyandra Ravi \& Anil } \\
\text { Hibiscus hispidissimus Griffith } \\
\text { H. molleriana } \\
\text { H. furcatus Roxb. } \\
\text { Kydia calycina Roxb. } \\
\text { Sida cordata (Burm.f.) Borssum } \\
\text { Sida cordata (Burm. f.) Borssum } \\
\text { Thespesia lampas (Cav.) Dalz. ex Dalz. \& Gibs. } \\
\text { Kydia calycina Roxb. }\end{array}$ & $\begin{array}{l}\text { Amazonia abutili Hosag. } \\
\text { Asteridiella julostylidis Hosag. \& Abraham } \\
\text { Irenopsis molleriana (Wint.) Stev. } \\
\text { Irenopsis mudumalaiensis Hosag. } \\
\text { Irenopsis sidae (Rehm) Hughes } \\
\text { Irenopsis sidae (Rehm) Hughes var. indica Hosag. \& Manoj. } \\
\text { Irenopsis thespesiae Hansf. } \\
\text { Meliola kydiae-calycinae Hansf. \& Thirum. }\end{array}$ \\
\hline 58. & Marantaceae 1 & Asteridiella & Schumannianthus virgatus (Roxb.) Rolfe & Asteridiella schumannianthi Hosag. et al. \\
\hline
\end{tabular}




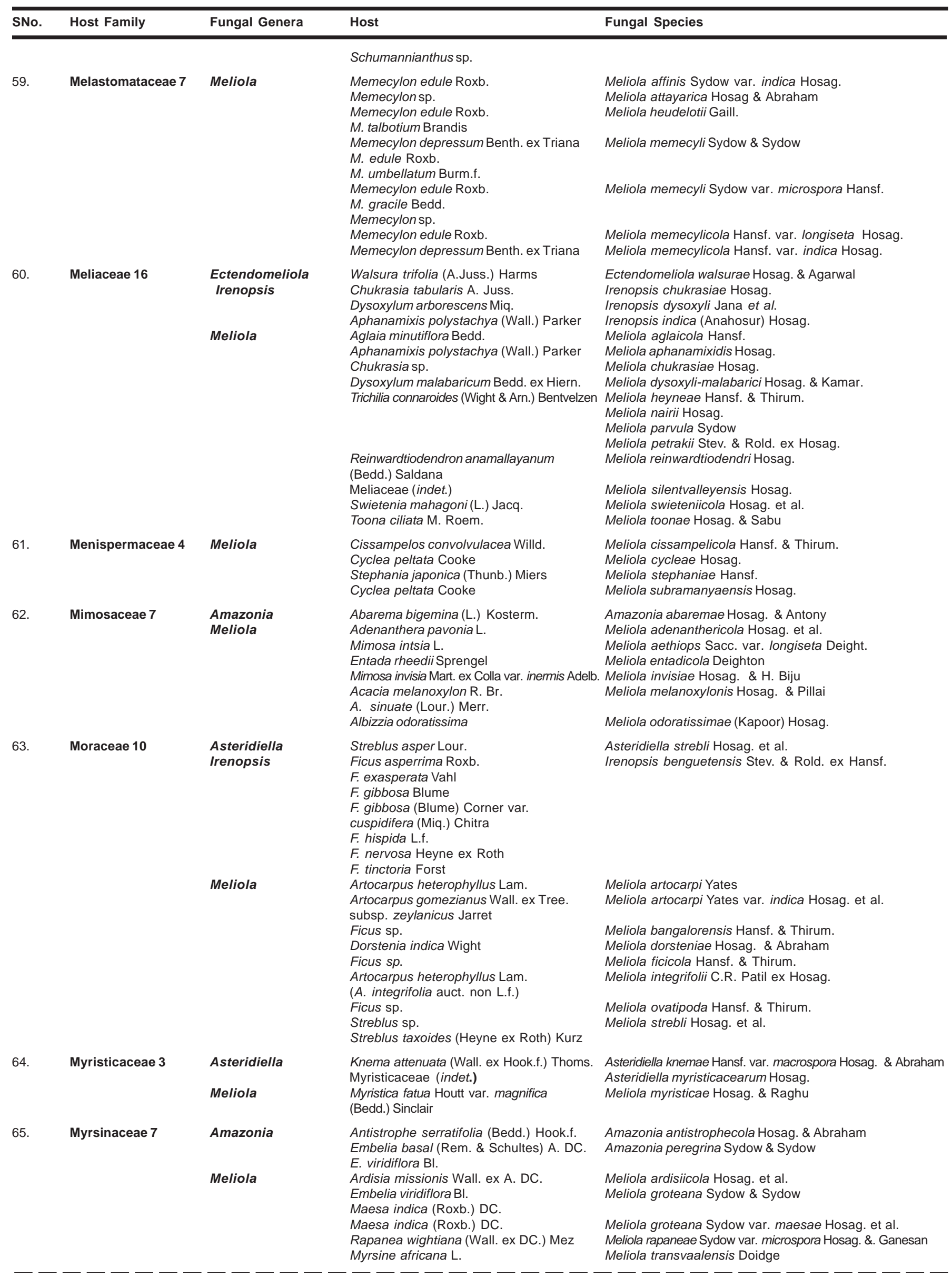




\begin{tabular}{|c|c|c|c|c|}
\hline SNo. & Host Family & Fungal Genera & Host & Fungal Species \\
\hline 66. & Myrtaceae 18 & $\begin{array}{l}\text { Amazonia } \\
\text { Asteridiella } \\
\text { Meliola }\end{array}$ & $\begin{array}{l}\text { Syzygium cumini Skeels } \\
\text { Syzygium sp. } \\
\text { Eugenia sp. } \\
\text { Eugenia fruticosa Roxb. } \\
\text { Syzygium claviflora Roxb. } \\
\text { Eugenia sp. } \\
\text { Syzygium laetum (Buch.- Ham.) Gandhi } \\
\text { S. munroni (Walp.) Chandr. } \\
\text { Eugenia floccosa Bedd. } \\
\text { Syzygium munroni (Walp.) Chandr. } \\
\text { Syzygium jambos (L.) Alst. } \\
\text { Eugenia stocksii Duthie } \\
\text { Eugenia eucalyptoides F. Muell. } \\
\text { Syzygium sp. } \\
\text { Syzygium zeylanicum (L.) DC. } \\
\text { Syzygium lanceolatum (Lam.) Wight \& Arn. } \\
\text { Syzygium laetum (Buch.-Ham.) Gandhi } \\
\text { Eugenia sp. } \\
\text { Syzygium sp. } \\
\text { Syzygium benthamianum (Wight ex } \\
\text { Duthie) Gamble } \\
\text { Psidium guajava L. }\end{array}$ & $\begin{array}{l}\text { Amazonia syzygii Hosag. } \\
\text { Asteridiella atricha (Speg.) Hansf. } \\
\text { Asteridiella eugeniae-fruticosae Jana et al. } \\
\text { Asteridiella ohiana (Stev.) Hansf. var. major Kar \& Maity } \\
\text { Meliola densa Cooke } \\
\text { Meliola eugeniae-jamboloidis Hansf. } \\
\text { Meliola eugeniae-jamboloidis Hansf. var. amphigena Kar \& Maity } \\
\text { Meliola eugeniae-stocksii } \\
\text { Hosag. } \\
\text { Meliola eugeniicola Stev. } \\
\text { Meliola gersoppaensis Hosag. et al. } \\
\text { Meliola laxa Gaill. var. Indica Hosag. et al. } \\
\text { Meliola maduraiensis Hosag. et al. } \\
\text { Meliola pulchella Speg. var. syzygii Hosag. } \\
\text { Meliola ranganathii Hansf. } \\
\text { Meliola syzygigena Hosag. \& Kamar. } \\
\text { Meliola syzygii-benthamianii Hosag. \& Abraham } \\
\text { Meliola trichostroma (Kunze) Toro }\end{array}$ \\
\hline 67. & Olacaceae 2 & Meliola & $\begin{array}{l}\text { Olax wightiana Wall. ex Wight \& Arn. } \\
\text { Strombosia ceylonica Gard. }\end{array}$ & $\begin{array}{l}\text { Meliola olacicola Hosag. } \\
\text { Meliola strombosiae Hosag. et al. }\end{array}$ \\
\hline 68. & Oleaceae 17 & $\begin{array}{l}\text { Irenopsis } \\
\text { Meliola }\end{array}$ & $\begin{array}{l}\text { Chionanthus mala-elengi (Dennst.) Green } \\
\text { (Linociera malabarica Wall. ex G. Don) } \\
\text { Olea dioica Roxb. } \\
\text { Ligustrum neilgherrense Wight } \\
\text { Jasminum sp. } \\
\text { Jasminum rottlerianum Wall. ex A. DC. } \\
\text { Jasminum flexile Vahl } \\
\text { J. malabaricum Wight } \\
\text { J. ozoricum L. } \\
\text { Olea glandulifera Wall. ex. G. Don. } \\
\text { Jasminum sp. } \\
\text { Jasminum sambac (L.) Ait. } \\
\text { Jasminum sp. } \\
\text { Jasminum auriculatum Vatil } \\
\text { J. rottlerianum Wall. ex A. DC. } \\
\text { Ligustrum walkeri Roxb. ssp. } \\
\text { walkeri (Decne) Green (Ligustrum } \\
\text { walkeri Decne) } \\
\text { Ligustrum perrottettii DC. } \\
\text { Chionanthus mala-elengi (Dennst.) } \\
\text { Green (Linociera malabarica Wall. ex Don) } \\
\text { Olea dioica Roxb. } \\
\text { Olea sp. } \\
\text { Ligustrum perrottetti DC. } \\
\text { Chionanthus ramiflora Roxb. } \\
\text { (Linociera intermedia Wight) } \\
\text { Chionanthus mala-elengi (Dennst.) Green } \\
\text { (Linociera malabarica Wall. ex G. Don) } \\
\text { Olea dioica Roxb. }\end{array}$ & $\begin{array}{l}\text { Meliola ligustricola Hosag. et al. } \\
\text { Meliola linocierae-malabaricae Hosag. } \\
\text { Meliola malabarensis Hansf. } \\
\text { Meliola mayapeae Stev. } \\
\text { Meliola mayapiicola Stev. var. indica Hosag. } \\
\text { Meliola oleacearum Hosag. }\end{array}$ \\
\hline 69. & Opiliaceae 5 & Meliola & $\begin{array}{l}\text { Cansjera rheedi Gmel. } \\
\text { Cansjera rheedii Gmel. } \\
\text { Cansjera rheedii Gmel. } \\
\text { Opilia amentacea Roxb. } \\
\text { Cansjera rheedi Gmel. } \\
\text { Lepionurus sylvestris DC. }\end{array}$ & $\begin{array}{l}\text { Meliola cansjerae Hansf. \& Thirum. } \\
\text { Meliola consjerae Hansf. \& Thirum. var. indica Hosag., } \\
\text { Siddappa \& Udaiyan } \\
\text { Meliola cansjericola Hosag. } \\
\text { Meliola opiliae Sydow } \\
\text { Meliola opiliae Sydow var. singalensis Hansf. }\end{array}$ \\
\hline 70. & Pandanaceae 2 & Meliola & $\begin{array}{l}\text { Pandanus sp. } \\
\text { Pandanus sp. }\end{array}$ & $\begin{array}{l}\text { Meliola kapoorii Hosag. \& P.A. Raghu } \\
\text { Meliola pandanacearum Hosag. \& Abraham }\end{array}$ \\
\hline 71. & Periplocaceae 3 & Meliola & $\begin{array}{l}\text { Hemidesmus indicus (L.) R.Br. } \\
\text { Hemidesmus indicus (L.) R. Br. } \\
\text { Hemidesmus indicus (L.) R. Br. }\end{array}$ & $\begin{array}{l}\text { Meliola hemidesmi Kamal \& Gupta } \\
\text { Meliola hemidesmicola Hosag. } \\
\text { Meliola hemidesmicola Hosag. var. indica Hosag. \& Manoj. }\end{array}$ \\
\hline 72. & Pinaceae 1 & Asteridiella & Taxus sp. & Asteridiella pitya (Sacc.) Hansf. \\
\hline 73. & Piperaceae 4 & Meliola & $\begin{array}{l}\text { Lepianthes umbellata (L.) Rafin } \\
\text { Piper trichostachyon (Miq.) DC. } \\
\text { Piper sp. } \\
\text { Piper nigrum L. } \\
\text { Piper nigrum L. }\end{array}$ & $\begin{array}{l}\text { Meliola lepianthedis Hosag. \& Kamar. } \\
\text { Meliola stenospora Wint. } \\
\text { Meliola stenospora Wint. var. major Hansf. } \\
\text { Meliola thetei Hosag. }\end{array}$ \\
\hline 74. & Pittosporaceae 1 & Meliola & Pittosporum dasycaulon Miq. & Meliola polytricha Kalch. \& Cooke \\
\hline
\end{tabular}




\begin{tabular}{|c|c|c|c|c|}
\hline SNo. & Host Family & Fungal Genera & Host & Fungal Species \\
\hline 75. & Poaceae 12 & Meliola & $\begin{array}{l}\text { Phragmatis karka (Retz.) Trin. ex Steud. } \\
\text { Cymbopogon flexuous (Nees ex Steud.) Wats. } \\
\text { C. nardus (L.) Rendle } \\
\text { Centotheca lappacea (L.) Desr. } \\
\text { Ischaemum zeylanicum Bor } \\
\text { Lophotherum gracile Brongn } \\
\text { Desmostachys bipinnata (L.) Stapf } \\
\text { Cyrtococcum longipes (Wight \& Arn. } \\
\text { Hook.f.) Camus } \\
\text { Vetiveria zizanoides (L.) Nash } \\
\text { Bambusa sp. } \\
\text { Sinarundinaria microphylla (Munro) } \\
\text { C.S. Chao \& Renvoize } \\
\text { Bambusa sp. } \\
\text { Saccharum spontaneum L. } \\
\text { Setaria sp. } \\
\text { Themeda cymbaria Hack. } \\
\text { Themeda triandra Forssk. }\end{array}$ & $\begin{array}{l}\text { Meliola arundinis Pat. } \\
\text { Meliola cymbopogonis Kapoor } \\
\text { Meliola panici Earle } \\
\text { Meliola panici Earle var. laciacidis (Toro) Hansf. } \\
\text { Meliola panici Earle var. macropodia Hosag. \& Abraham } \\
\text { Meliola panici Earle var. vetiveriicola Gawande, Agarwal \& Hosag. } \\
\text { Meliola phyllostachydis Yamam. } \\
\text { Meliola phyllostachydis Yamam. var. microspora Hosag. et al. } \\
\text { Meliola sacchari Sydow } \\
\text { Meliola setariae Hansf. \& Deight. var. indica Patil \& Mahamu. } \\
\text { Meliola themedae Stev. \& Rold. ex Hansf. var. indica Hosag. } \\
\text { Meliola themedicola Hosag. et al. }\end{array}$ \\
\hline 76. & Polygonaceae 2 & Meliola & $\begin{array}{l}\text { Polygonum chinense L. } \\
\text { Polygonum chinense L. }\end{array}$ & $\begin{array}{l}\text { Meliola polygoni Srinivasulu } \\
\text { Meliola polygonicola Hosag. }\end{array}$ \\
\hline 77. & Rhamnaceae 4 & $\begin{array}{l}\text { Amazonia } \\
\text { Appendiculella } \\
\text { Irenopsis } \\
\text { Meliola }\end{array}$ & $\begin{array}{l}\text { Gouania microcarpa DC. } \\
\text { Hovenia dulcis Thumb. } \\
\text { Gouania leptostachya DC. } \\
\text { Ziziphus glabrata Heyne ex Roth } \\
\text { (Z. trinervia Roxb.) } \\
\text { Z. rugosa Lamk. } \\
\text { Z. xylopyrus (Retz.) Willd. }\end{array}$ & $\begin{array}{l}\text { Amazonia gouaniae Hosag. \& Braun } \\
\text { Appendiculella hoveniae Kar \& Maity } \\
\text { Irenopsis tenuissima (Stev.) Stev. var. major Kar \& Maity } \\
\text { Meliola ziziphi Hansf. \& Thirum. }\end{array}$ \\
\hline 78. & Rhizophoraceae 1 & Meliola & Carallia integrerrhima DC. & Meliola anisophylleae Hansf. \& Deighton var. caralliae Hosag. et al. \\
\hline 79. & Rosaceae 7 & $\begin{array}{l}\text { Asteridiella } \\
\text { Irenopsis } \\
\text { Meliola }\end{array}$ & $\begin{array}{l}\text { Crataegus crenulata Roxb. } \\
\text { Rubus calycinus Don. } \\
\text { R. ellipticus Smith } \\
\text { R. vulgaris Meikle } \\
\text { Rubus sp. } \\
\text { Rubus moluccanus sensu Hook.f. } \\
\text { Pyracantha crenulata Roem. } \\
\text { Rubus ellipticus Smith } \\
\text { Rubus sp. } \\
\text { Rubus niveus Thunb. } \\
\text { Rubus micropetalus Gardn. } \\
\text { R. ellipticus Smith } \\
\text { Rubus sp. }\end{array}$ & $\begin{array}{l}\text { Asteridiella pygei Hansf. var. microspora Hosag. } \\
\text { Irenopsis rubi Patil \& Maham. } \\
\text { Meliola rubi Stev. \& Rold. ex Hansf. var. garhwalensis } \\
\text { (Srivastava \& Topal) Hosag. \& Balakr. } \\
\text { Meliola rubiella Hansf. } \\
\text { Meliola rubiella Hansf. var. indica Hosag. } \\
\text { Prataprajella rubi Hosag. et al. }\end{array}$ \\
\hline 80. & Rubiaceae 40 & $\begin{array}{l}\text { Appendiculella } \\
\text { Asteridiella } \\
\text { Meliola }\end{array}$ & $\begin{array}{l}\text { Psychotria flavida Talbot } \\
\text { Canthium sp. } \\
\text { Psychotria sp. } \\
\text { Psychotria globicephala Gamble } \\
\text { Saprosma corymbosum (Bedd.) Bedd. } \\
\text { Psychotria sp. } \\
\text { Rubiaceae (indet.) } \\
\text { Wendlandia scabra Kurz. } \\
\text { Wendlandia notianiana Wall. } \\
\text { Canthium angustifolium Roxb. } \\
\text { Mussaenda belilla Buch.- Ham. } \\
\text { (M. laxa Hook.f.) Hutch. ex Gamble } \\
\text { Neolamarkia cadamba (Roxb.) Bosser } \\
\text { Canthium parviflorum Lam. } \\
\text { (Plectronia parviflora Bedd.) } \\
\text { C. rheedii DC. } \\
\text { Canthium angustifolium Roxb. } \\
\text { Canthium rheedii DC. } \\
\text { Ixora parviflora Vahl } \\
\text { Chassalia sp. } \\
\text { Canthium parviflorum Lam. } \\
\text { C. rheedii DC. } \\
\text { Canthium rheedii DC. } \\
\text { Hedyotis stylosa R. Br. ex. Wight \& Arn. } \\
\text { Psychotria sp. } \\
\text { Canthium wightii } \\
\text { Ixora polyantha Wight } \\
\text { Ixora sp. } \\
\text { Ixora coccinea L. } \\
\text { I. elongata Heyne } \\
\text { I. nigricans R. Br. } \\
\text { Ixora coccinea L. } \\
\text { Ixora sp. }\end{array}$ & $\begin{array}{l}\text { Amazonia goosii Hosag. \& Abraham var. microspora Hosag. et al. } \\
\text { Amazonia psychotriae (Henn.) Theiss. } \\
\text { Amazonia psychotriae (P. Henn.) Theiss. var. macrospora } \\
\text { Hosag. et al. } \\
\text { Appendiculella wendlandiae Jana et al. } \\
\text { Asteridiella tarlacence Petrak } \\
\text { Meliola africana Hansf } \\
\text { Meliola anceps Sydow \& Sydow } \\
\text { Meliola cadambae Hosag. \& C.K. Biju } \\
\text { Meliola canthii Hansf. } \\
\text { Meliola canthii-angustifolii Hosag. } \\
\text { Meliola canthiicola Hosag. et al. } \\
\text { Meliola chandolensis C.R. Patil ex Hosag. } \\
\text { Meliola chassaliicola Hosag. } \\
\text { Meliola henryi Hosag. } \\
\text { Meliola henryi Hosag. var. oldenlandiae Hosag. et al. } \\
\text { Meliola hydei Hosag. et al. } \\
\text { Meliola ixorae Yates }\end{array}$ \\
\hline
\end{tabular}




\begin{tabular}{|c|c|c|c|c|}
\hline SNo. & Host Family & Fungal Genera & Host & Fungal Species \\
\hline & & & $\begin{array}{l}\text { Psychotria macrocarpa Hook.f. } \\
\text { Ixora coccinea L. } \\
\text { Hedyotis albo-nervia Bedd. } \\
\text { H. gamblei Henry \& Subram. } \\
\text { Knoxia sp. } \\
\text { Knoxia sumatrensis (Retz.) DC. } \\
\text { Knoxia sp. } \\
\text { Canthium dicoccum (Gaertn.) Teijsm. \& Binn. } \\
\text { var. umbellata (Wight) Sant. \& Merch. } \\
\text { Mitragyna parviflora Korth } \\
\text { Mitragyna tubulosa (Arn.) Hav. } \\
\text { Neanotis monosperma (Wall. ex Wight } \\
\text { \& Arn.) Lewis } \\
\text { Hedyotis sp. } \\
\text { Hedyotis sp. } \\
\text { Morinda umbellata L. } \\
\text { Canthium dicoccum (Gaertn.) Teys \& Benn. } \\
\text { (Plectronia umbellate Benth. \& Hook.) } \\
\text { Canthium rheedii DC. } \\
\text { Pavetta indica L. } \\
\text { Ixora coccinea L. } \\
\text { Psychotria nudiflora Wight \& Arn. } \\
\text { Ixora brachiata Roxb. ex DC. } \\
\text { I. coccinea L. } \\
\text { Ixora elongata Heyne ex G. Don. } \\
\text { Ixora arborea Roxb. ex Smith } \\
\text { Ixora coccinae L. } \\
\text { Tarenna asiatica (L.) Sant. \& Merch. } \\
\text { (Chomelia asiatica O. Kze.) } \\
\text { Webera corymbose (Willd.) } \\
\text { Wendlandia notoniana Wall. W. tomentosa }\end{array}$ & $\begin{array}{l}\text { Meliola ixorae-coccineae Hosag. \& Pillai } \\
\text { Meliola kanniyakumariana Hosag. } \\
\text { Meliola kanniyakumariana Hosag. var. brahmagiriense Hosag. et al. } \\
\text { Meliola knoxiicola Hosag. et al. } \\
\text { Meliola longiseta Hoehnel } \\
\text { Meliola mitragynae Sydow } \\
\text { Meliola mitragynae-tubulosae Hosag. \& Manoj. } \\
\text { Meliola neanotidis Hosag. et al. } \\
\text { Meliola oldenlandiae Hansf. \& Stev. } \\
\text { Meliola oldenlandiae Hansf. \& Stev. var. indica Hosag. et al. } \\
\text { Meliola palawanensis Sydow } \\
\text { Meliola plectroniae Hansf. } \\
\text { Meliola psychotriae Earle } \\
\text { Meliola psychotriae-nudiflorae Hosag. } \\
\text { Meliola randiicola Hansf. } \\
\text { Meliola ravii Hosag. et al. } \\
\text { Meliola tawaoensis Hansf. } \\
\text { Meliola twaitesiana Hansf. } \\
\text { Meliola weberae Kapoor }\end{array}$ \\
\hline 81. & Rutaceae 26 & $\begin{array}{l}\text { Irenopsis } \\
\text { Meliola }\end{array}$ & $\begin{array}{l}\text { Acronychia pedunculata (L.) Miq. } \\
\text { Melicope lunuankenda (Gaertn.) T.G. Hartley } \\
\text { (Euodia lunuankenda) (Gaertn.) Merr.) } \\
\text { Acronychia pedunculata (L.) Miq. } \\
\text { Glycosmis pentaphylla (Retz.) DC. } \\
\text { (G. cochinchinensis sensu Gamble) } \\
\text { Murraya koenigii (L.) Spreng. } \\
\text { Atalantia wightii Tanaka } \\
\text { Citrus aurantifolia (Christm.) Swingle } \\
\text { (C. medica L. var. acida (Roxb.) Hook. f.) } \\
\text { Citrus sp. } \\
\text { Toddalia sp. } \\
\text { Glycosmis mauritiana (Lam.) Tanaka } \\
\text { (G. pentaphylla Correa) } \\
\text { G. macrocarpa Wight } \\
\text { Toddalia asiatica (L.) Lam. } \\
\text { Citrus aurantium L. } \\
\text { Clausena indica (Dalz.) Oliver } \\
\text { C. dentate (Willd.) M. Roem. } \\
\text { Toddalia sp. } \\
\text { Luvunga sermentosa (Blume) Kurz } \\
\text { (L. elutherandra Dalz.) } \\
\text { Evodia luna-ankenda (Gaertn.) Merr. } \\
\text { Vepris bilocularis (Wight \& Arn.) Engl. } \\
\text { Paramignya armata (Thw.) Oliver } \\
\text { P. monophylla } \\
\text { Zanthoxylum ovalifolium Wight } \\
\text { Z. ovata Wight } \\
\text { Toddalia asiatica (L.) Lam. } \\
\text { Murraya paniculata (L.) Jack (M. exotica L.) } \\
\text { Atalantia monophylla (L.) Correa } \\
\text { Atalantia monophylla (L.) Correa } \\
\text { Pamburus missionis (Wight) Swingle } \\
\text { Toddalia asiatica (L.) Lam. (T. aculeate Pers.) } \\
\text { Vepris bilocularis (Wight \& Arn.) Engl. } \\
\text { Zanthoxylum ovalifolium Wight } \\
\text { Z. tetraspermum Wight \& Arn. } \\
\text { Zanthoxylum ovalifolium Wight } \\
\text { (a) }\end{array}$ & $\begin{array}{l}\text { Meliola cadigensis Yates var. toddaliae Hosag. et al. } \\
\text { Meliola cadigensis Yates var. glycosmidis (Kapoor) Hosag. } \\
\text { Meliola cannonicola Hosag. \& C.K. Biju } \\
\text { Meliola citricola Sydow \& Sydow } \\
\text { Meliola clausenae Hosag. } \\
\text { Meliola devikulamensis Hosag., H. Biju \& A. Manoj Kumar } \\
\text { Meliola luvungae Hosag. } \\
\text { Meliola macropoda Sydow } \\
\text { Meliola paramignyae Hosag. } \\
\text { Meliola rickiana Hansf. var. zanthoxyli Hosag. } \\
\text { Meliola tecleae Hansf. var. toddaliae-asiaticae Hansf. } \\
\text { Meliola tenella Pat. } \\
\text { Meliola tenella Pat. var. atalantiae (Pat.) Hansf. } \\
\text { Meliola tenella Pat. var. atalantiicola Hosag. } \\
\text { Meliola toddaliae Doidge } \\
\text { Meliola toddaliicola Hansf. var. indica Hansf. \& Thirum. } \\
\text { Meliola vepridis Hosag. et al. } \\
\text { Meliola zanthoxyli Hansf. } \\
\text { Meliola zanthoxyli-ovalifolii Hosag. }\end{array}$ \\
\hline 82. & Sabiaceae 1 & Asteridiella & Meliosma simplicifolia (Roxb.) Walp. & Asteridiella meliosmae Kar \& Maity \\
\hline 83. & Santalaceae 3 & Meliola & $\begin{array}{l}\text { Osyris arborea Wall. } \\
\text { Osyris quadriseptata Salz. ex Decne } \\
\text { Scleropyrum pentandrum (Dennst.) } \\
\text { Mabberley (S. wallichianum Wight \& Arn.) }\end{array}$ & $\begin{array}{l}\text { Meliola osyridicola Hansf. } \\
\text { Meliola osyridicola Hansf. var. indica Hosag. } \\
\text { Meliola scleropyri Hosag. }\end{array}$ \\
\hline 84. & Sapindaceae 18 & $\begin{array}{l}\text { Irenopsis } \\
\text { Meliola }\end{array}$ & $\begin{array}{l}\text { Nephelium longan Lour. } \\
\text { Allophylus serrulatus Radlk. } \\
\text { Allophyllus concanicus Radlk. var. }\end{array}$ & $\begin{array}{l}\text { Irenopsis nephelii Patil \& Maham. } \\
\text { Meliola allophyligena Hosag. } \\
\text { Meliola allophyli-concanici Hosag. }\end{array}$ \\
\hline
\end{tabular}




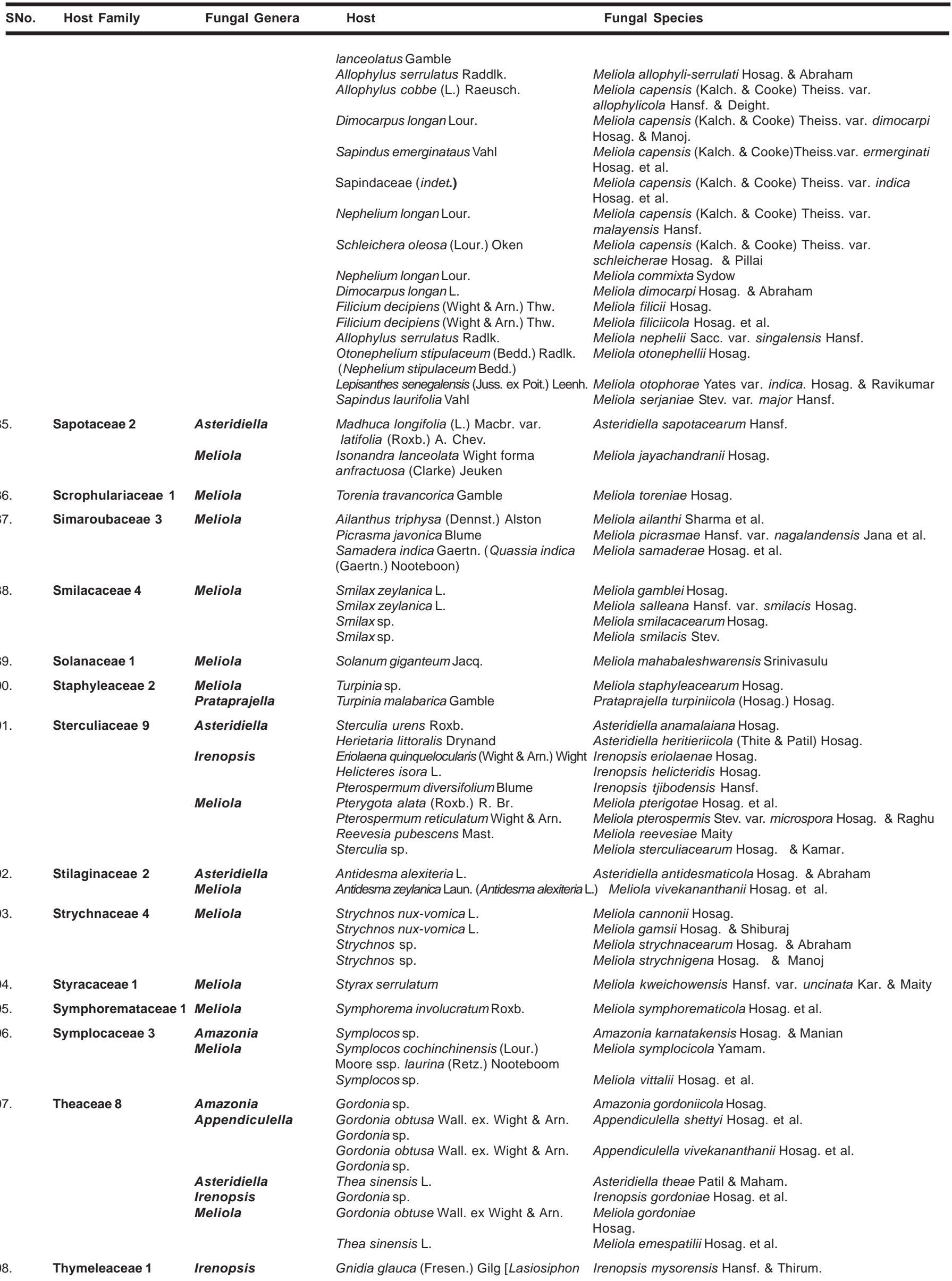




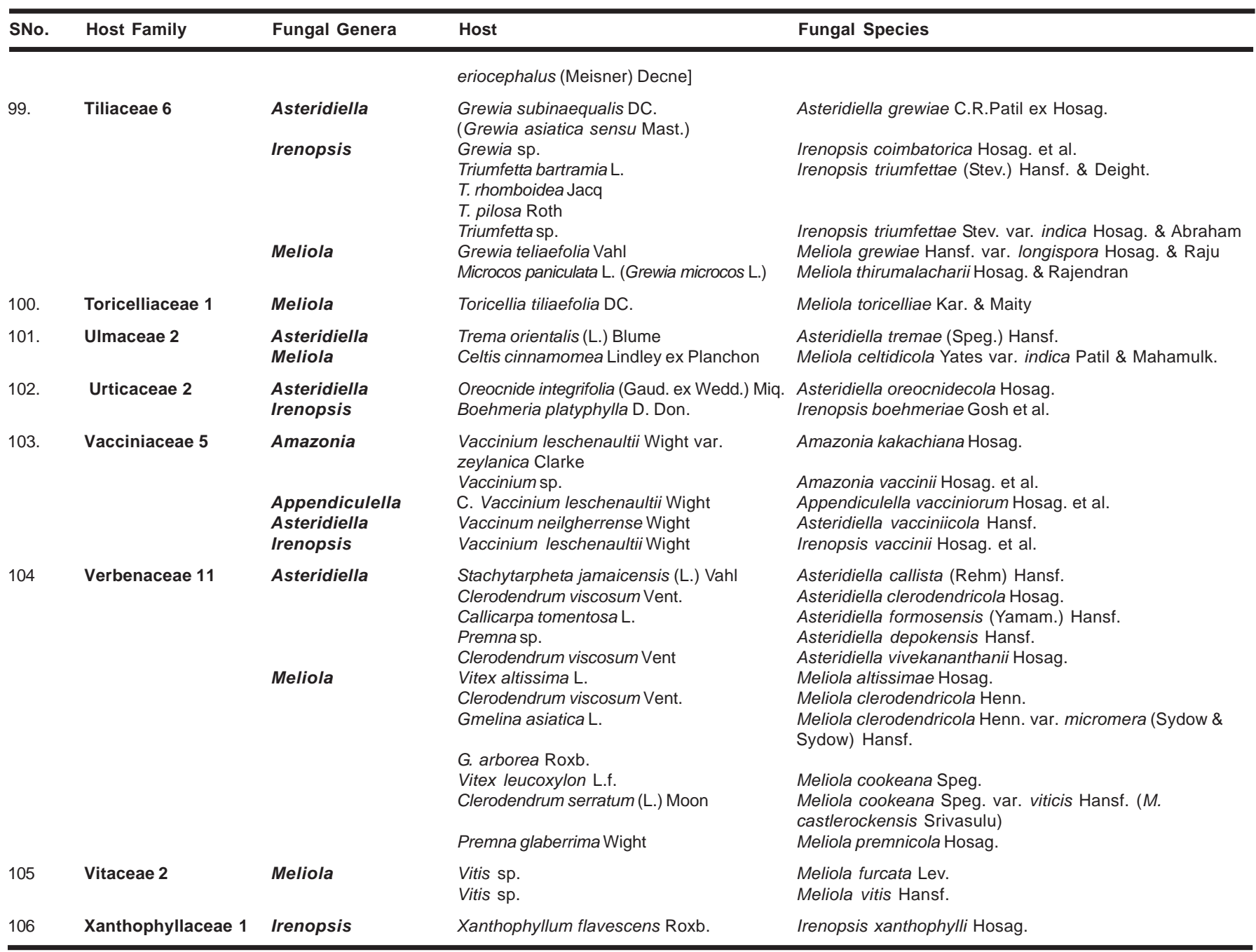

Verbenaceae and Asclepiadaceae (11 taxa each), Moraceae (10 taxa). Rest of the families represent less than this number. This reveals that the members of these families are more compatible or susceptible to the black mildew fungi than other members.

Of the ten biogeographical regions of India, Trans Himalayan, Desert, Semi Arid and Coastal vegetation (Mangroves) are devoid of these fungi. The Western Ghats and North-East India are very rich in biodiversity. In proportion to 4000 Phanerogams in the Western Ghats, more than 400 meliolas are known. However, from north-east India, only 6 are known. No doubt this region also must be rich in meliolas and the poor representation could be due to inadequate survey and taxonomic study of the group in the area.

These fungi infect variety of plants, viz. exotics, introduced for the aforestation programmes (Hosagoudar et al. 2002; Archana \& Hosagoudar 2006), plantation crops (Hosagoudar \& Abraham 2000), medicinal plants (Hosagoudar 2003), endemics (Hosagoudar 2003), economically important plants, plants of non-wood forest produce, wild edible plants, commercially timber yielding plants (Hosagoudar 2006 a,b,c, etc.). The study of the occurrence of these fungi on the sacred groves has also been conducted as a case of reference (Hosagoudar et al. 2005).

These fungi infect both wild and cultivated plants but more on wild. However, the infection is also noticed on wild relatives of present day's cultivars viz. Vigna spp., Mangifera andamanica, etc. These fungi are host specific but still there is a both specific and generic host range.

Most of the fungi are restricted in their distribution to their biogeographical regions. However, Asteridiella malloti (Hansf. \& Thirum.) Hansf., Meliola bauhiniicola Yamam, M. chandleri Hansf., M. heudelotii Gaill., M. maredumilliana Hosag. \& Mohanan, M. mayapeae Stev. and, M. opiliae Sydow are known from the Western Ghats and Eastern Ghats (Deccan Plateau) in the peninsular India; Meliola butleri Sydow, M. malacotricha Speg., M. mitragynae Sydow and M. tabernaemontanicola Hansf. \& Thirum. are known from Western Ghats and Himalayan region, Meliola simillima Ellis \& Everh. var. major Hansf. and M. tawaoensis Hansf. are common to Eastern Ghats and Himalayan region; Meliola mangiferae Earle is common to Western Ghats and Andaman Islands. This indicates that although endemism is very prevalent among these, there are, however some with wide geographical distribution.

These fungi are predominantly on forest plants but are not uncommon on plantation crops like Mango, Cinnamomum, Cashew, Wattles, etc. (Hosagoudar \& Balakrishnan 1995; Hosagoudar \& Abraham 2000).

A maiden venture on certain pockets of the forest plants yielded several novelties. Hence, the nature convinces us that we are in the beginning stage in understanding of the microbes. 


\section{Key to the families of the order meliolales}

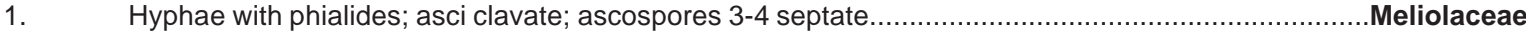

1. Hyphae without phialides; asci cylindrical to sub cylindrical, ascospores 1-2 septate Armatellaceae

\section{Key to the genera of the family armatellaceae}

1. Ascospores uniseptate at maturity, septum almost median.

Armatella

1. Ascospores 2-septate at maturity, septa at the distal ends Basavamyces

\section{Key to the genera of the family meliolaceae}

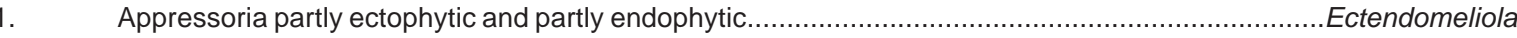

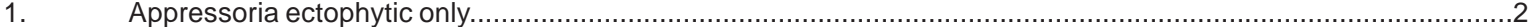

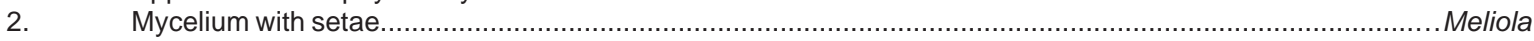

Mycelium without setae

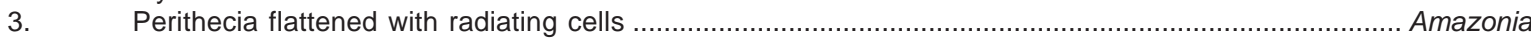

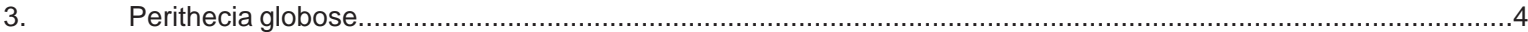

4. Perithecial setae, repent setae and appendages present.

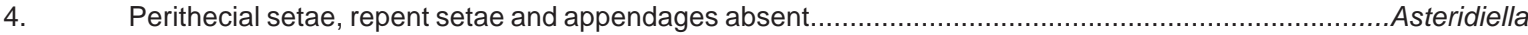

5. Perithecial setae present but devoid of repent setae and appendages..............................................renopsis

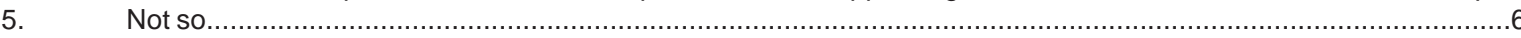

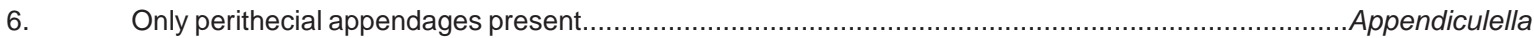

6. Both perithecial appendages and repent setae present. ..Prataprajella

\section{References}

Archana, G.R. \& V.B. Hosagoudar (2006). Black mildew disease on wattles (Acacia spp.) in Kerala state. National Conference on Recent Trends in Mycological Research, Dec. 28th \& 29, J.J College of Arts and Sci. Pudukottai, TN, p. 85.

Hosagoudar, V.B. \& T.K. Abraham (2000). Black mildews (Meliolaceae) on Plantation crops. Journal of Mycopathological Research 38: 1-6.

Hosagoudar, V.B., T.K. Abraham \& S. Shiburaj (2002). Black mildew disease on Acacia species in India. Journal of Mycopathological Research 40: $45-47$.

Hosagoudar, V.B. (2003). Meliolaceous fungi on rare medicinal plants in southern India. Zoos' Print Journal 18: 1147-1154.

Hosagoudar, V.B. (2003). Endemic Meliolas and Meliolas on Endemic plants in Western Ghats, India. Zoos' Print Journal 18: 1243-1252.

Hosagoudar, V.B. \& H. Biju (2003). Host range of Meliola jasmini Hansf. \& Stev. New Botanist 30: 153-162.

Hosagoudar, V.B., H. Biju \& K.A.Anu Appaiah (2005). Foliar fungal parasites of Western Ghats found on the plants of sacred groves in Dakshina Kannada and Udupi districts of Karnataka State. Journal of Mycopathological Research 43: 203-209.

Hosagoudar, V.B. (2006). Meliolaceous fungi on economically important plants in India-II: on plants of non-wood forest produce. Zoos' Print Journal 21: 2356-2371.

Hosagoudar, V.B. (2006). Meliolaceous fungi on economically important plants in India-III: on wild edible plants. Zoos' Print Journal. 21:24252438 .
Hosagoudar, V.B. (2006). Meliolaceous fungi on an economically important plants in India-I. on commercially timber yielding plants. Journal of Economic E Taxonomic Botany 30: 818-850.

Hosagoudar, V.B. (2006). Biogeographical distribution of Meliolaceae members in India. Zoos' Print Journal 21: 2495-2505.

Hosagoudar, V.B. \& H. Biju (2006). Studies on foliicolous fungi - XXII. Microfungi of Silent Valley National Park, Palghat district in Kerala State. Journal of Mycopathological Research 44: 39-48.

Hosagoudar, V.B., H. Biju \& K.A. Anu Appaiah (2006). Studies on foliicolous fungi - XX. Microfungi of Coorg, Karnataka. Journal of Mycopathological Research 44: 1-25.

Karthikeyan, S. (2000). Statistical analyses of flowering plants of India. In: Singh, N.P., Singh, D.K., Hajra, P.K. and Sharma, B.D. (eds.). Flora of India. Introductiory vol. II. 201-217. New Delhi.

Mudgal, V. \& P.K. Hajra (1997-99). Floristic diversity and conservation strategies in India. Botanical Survey of India, Dehra Dun, Vol. I\&II.

Sharma, J.R., V. Mudgal \& P.K. Hajra (1997). Floristic diversity and conservation strategies in India. Cryptogams and Gymnosperms I. Botanical Survey of India, Dehra Dun. 